\title{
Modeling on Actuation Behavior of Macro-Fiber Composite Laminated Structures Based on Sinusoidal Shear Deformation Theory
}

\author{
Jiarui Zhang ${ }^{1}{ }^{\oplus}$, Jianwei $\mathrm{Tu}^{1, *}, \mathrm{Zhao} \mathrm{Li}^{1}{ }^{1}, \mathrm{Kui} \mathrm{Gao}^{1}$ and Hua Xie ${ }^{1,2}$ \\ 1 Hubei Key Laboratory of Roadway Bridge and Structure Engineering, Wuhan University of Technology, \\ Wuhan 430070, China \\ 2 China Construction Third Engineering Bureau Co., Ltd., Wuhan 430070, China \\ * Correspondence: tujianwei@whut.edu.cn
}

Received: 13 June 2019; Accepted: 16 July 2019; Published: 19 July 2019

check for updates

\begin{abstract}
A new piezoelectric composite, macro fiber composite (MFC) is recombined with piezoceramic fibers, an epoxy resin basal body, and an interdigitated electrode. It has been widely applied in vibration reduction and deformation control of thin-walled structures, due to its great deformability and flexibility. Research on its actuation performance is mostly concentrated on the MFC actuating force calculation based on classical plate theory (CPT), and the overall modeling of MFC and its structure. However, they have some deficiencies in the tedious calculating process and neglect of shear deformation, respectively. To obtain a precise MFC actuating force, the sinusoidal shear deformation theory (SSDT) is adopted to deduce the MFC actuating force formula, and global-local displacement distribution functions are introduced to help the MFC laminated plate structure satisfy the deformation compatibility and stress balance. For instance, in the end displacement calculation of the MFC laminated beam structure. The experimental result of the MFC laminated beam is compared with those of the MFC actuating force based on SSDT and on CPT, which indicates that the MFC actuating force formula based on SSDT can reach higher computational accuracy.
\end{abstract}

Keywords: macro fiber composite; MFC laminated structure; sinusoidal shear deformation theory

\section{Introduction}

As a widely used piezoelectric smart material, piezoceramics can have an actuating performance through the inverse piezoelectric effect and a sensing performance through the direct piezoelectric effect. They are often applied in areas such as structural vibration reduction, energy harvesting, and health monitoring, etc. [1-3]. Piezoceramics are characterized by their high sensitivity and good thermal stability, albeit poor flexibility. Therefore, the National Aeronautics and Space Administration (NASA) lab in Langley has developed a new type of piezoelectric smart material, macro fiber composite (MFC) [4], which is recombined with piezoceramic fiber rods with rectangular sections, an epoxy resin basal body, a copper electrode, and a polymer film. With a higher electromechanical transformation efficiency and better flexibility, MFC has wide application prospects and research value.

In the vibration reduction of thin-walled structures and deformation control, MFC has received extensive attention. In the study of controlling the vibration response of thin-walled structures with MFC, its actuating force can be obtained by two methods: (i) overall modeling is made for the MFC and the structure, then voltage directly serves as the input to perform a simulating calculation for the structure; (ii) or the MFC is equivalent to the external actuating force to be exerted on the structure for the simulating calculation. With regard to the former, Dano and Jullière [5] studied MFC's active control over thermally-induced deformation of the composite plate, performed finite element modeling 
of the MFC and flat plate construction with ABAQUS finite element software, obtained the actuating force of the MFC by the finite element method, proposed an active closed-loop control algorithm, and verified the feasibility of the MFC control over thermally-induced deformation. Kim et al. [6] have performed overall modeling for the MFC and shell structure, and then solved the actuating force of the MFC by the variation principle and finite element discretization. On this basis, they undertook a vibration reduction simulation and experimental analysis for an end-sealing shell structure attached to the MFC with a negative velocity feedback method. Through overall modeling of the MFC and thin-walled cylindrical structure, Sohn et al. $[7,8]$ calculated the actuating force of the MFC by using the Donnell-Mushtari shell theory and Lagrange equation, as well as the variational principle and finite element discretization, respectively. Based on this, they have studied the vibration control effect of the cylindrical structure with the MFC in air and in water, which proved that the MFC can effectively reduce vibrations in both conditions. Zhang et al. [9] performed a finite element analysis on the MFC integrated plate structure with different fiber orientations, established the linear electromechanical coupling model of the MFC integrated plate-shell structure, analyzed the actuating force of the MFC by variational principle and finite element discretization, and performed contrastive analysis between the simulation results and the experimental results. Aiming for vibrational control of flexible structures, Wang et al. [10] studied the optimal configuration of the MFC actuator, established a model for MFC integrated plate-shell structures with the finite element method, and obtained the actuating force of the MFC by the Hamilton's principle. On this basis, they provided the best location and fiber orientation for MFCs in different working conditions. Zhou et al. [11] have studied the aeroelastic stability of composite plates embedded within MFCs in supersonic flows, and established the dynamical equation of MFC and plate structure through virtual work principle, and gave the actuating force expression of the MFC, which indicates that both MFC lamination angles and temperature variation will produce remarkable influences on the aeroelastic stability boundary of curved composite plates in supersonic airflows.

Accurate simulation results can be obtained by overall modeling of the MFC and structure. However, this is hard to implement for complicated structures. With regard to the structure, the mass of the MFC slice is negligible and produces little influence on the structural mode. As a result, the MFC is equivalent to the external actuating force to be exerted onto the structure, which is a simpler and more reasonable method. Gao et al. [12] arranged MFCs on vertical fin structures to deduce the MFC equivalent actuating bending moment equation and the sensing voltage equation by utilizing the classical plate theory (CPT), and accomplished the simulation and experiment of vibrational control of vertical fin structures based on negative feedback by establishing a simplified model of the vertical fin structures attached to the MFC with ABAQUS finite element software, proving that MFCs have a great controlling effect on the vertical fin structures. Li et al. [13] studied the equivalent actuating force based on the elastic deformation theory under the condition of partial abhesion of the MFC, analyzed the influence of the percentage and location of delamination on actuating performance, and verified the experimental result. Ma et al. [14] studied MFC's active control over a cantilever beam structure in axial motion, calculated the actuating force of the MFC by CPT, and used the MFC as an equivalent actuating force to be exerted onto the cantilever beam structure, proving that the MFC can effectively restrain vibration of the cantilever beam structure. Dong et al. [15] modeled a plate structure integrated with a piezoelectric actuator in a semi-analytical fashion, and applied the analytical equivalent force to the finite element models. The equivalent force is deduced from the bending effort balance between the equivalent loads, and is verified by the actuating experiment of composite laminates integrated with the P1 type of MFC. The experimental results were in good agreement with the simulation results. Sun et al. [16] designed an active serrated microflap based on an MFC to reduce the fatigue load of wind turbine blades, obtained the actuating force of the MFC by CPT and the variation principle, and established a feedback control system suitable for the microflap by making MFC equivalent to the actuating force, indicating that this active serrated microflap can effectively reduce fatigue load. At present, the calculating methods of the MFC equivalent actuating force have mostly been concentrated 
on considering the bending deformation and tensile deformation of the MFC integrated plate structure, but ignored the influence of shear deformation. Aiming for piezoceramics, Crawley and Luis [17] studied the actuating force and actuating bending moment formulas considering shear deformation of the stickup layer and provided a distribution curve of piezoceramic's actuating force to simulate the actuating performance of the piezoelectric material well. Thereafter, Crawley and Anderson [18] studied the actuating force formula of a piezoelectric material considering bending deformation, but not considering shear deformation. Sonti et al. [19] took uniformly polarized piezoelectric actuators with different shapes as the study objects. By analyzing their equivalent forces and wavenumber spectrum, the influence of the shape of the piezoelectric actuators on the modal response of flat plates is studied, and the general expression of equivalent force of piezoelectric actuators was deduced by the small deflection theory. The results show that the uniformly polarized piezoelectric actuator has a high order mode coupling trend regardless of the shape of the piezoelectric actuator. Deraemaeker et al. [20] deduced the analytical expressions of equivalent forces of an orthotropic piezoelectric actuator based on the small deflection theory under general conditions by the Hamilton's principle and two different mathematical methods. Both methods obtained the same analytical expressions of equivalent forces. Since the thickness of the stickup layer is much smaller than that of the actuator and structure, Im and Atluri [21] perfected this calculation method, ignoring the influence of the stickup layer, and deduced the complete piezoceramic actuating force formula with higher accuracy by further considering the influence of beam shear deformation on shear stress between the piezoelectric material and the beam structure. It is indicated that a better actuating force formula can be obtained by considering shear deformation of the piezoelectric material and the structure. Therefore, the MFC actuating force needs to consider shear deformation of the MFC and the structure.

With regard to laminated plate structures, it is hard to obtain a precise stress-strain result by only considering the bending deformation and tensile deformation. Shear deformation is taken into account for a more precise result [22-24]. The stickup position of MFC integrated plate-shell structures can be treated as a bistratal laminated plate structure, so the shear deformation theory can be applied into the actuating force formula calculation of MFC integrated plate structures. Because the sinusoidal shear deformation theory (SSDT) can precisely simulate the deformation and internal force calculation of laminated plate structures $[25,26]$, this paper introduces a local displacement distribution function with the SSDT, proposes a new MFC actuating force formula, and performs contrastive analysis between the new formula and the one based on classical plate theory (CPT) $[27,28]$. To make a detailed explanation of the applicability of the MFC actuating force formula, the MFC laminated beam structure in [29] is exemplified to analyze the MFC internal force distribution and free end displacement in different working conditions, which proves the applicability of the theory in this paper.

\section{The Constitutive Relation of MFC}

The P1 type and P2 type MFCs have been chosen to be the study objects. The polarization direction of the former is parallel to the fiber orientation, while that of the latter is parallel to through-thickness direction, as is shown in Figure 1. The constitutive relation of the MFC is [30,31]:

$$
\begin{aligned}
\varepsilon_{i} & =s_{i j}^{E_{j}} \sigma_{j}+d_{m i} E_{m} \\
D_{m} & =d_{m i} \sigma_{i}+\kappa_{i m}^{\sigma} E_{m}
\end{aligned},
$$

where $s_{i j}^{E}$ represents the flexible coefficient when the electric field intensity is constant, $i$ is the strain direction of the MFC, and $j$ is the stress direction of MFC; $d_{m i}$ represents the piezoelectric strain coefficient, $m$ is the electric field action direction; $D_{m}$ represents the electric displacement in the $i$ direction; $\kappa_{i m}^{\sigma}$ is the dielectric constant when stress is constant; $E_{m}$ is the electric field intensity exerted in the $m$ direction. 


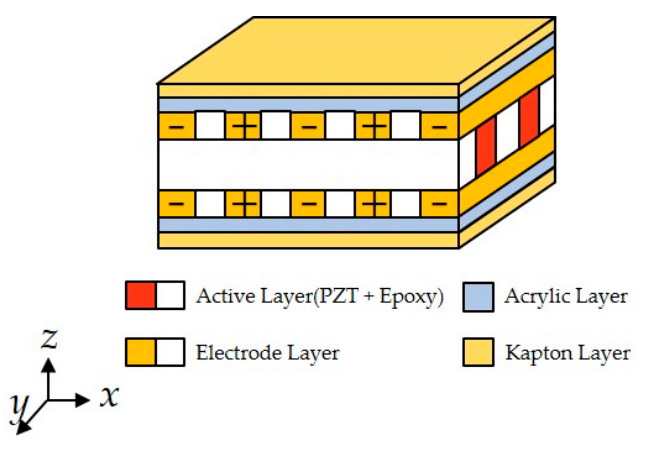

(a)
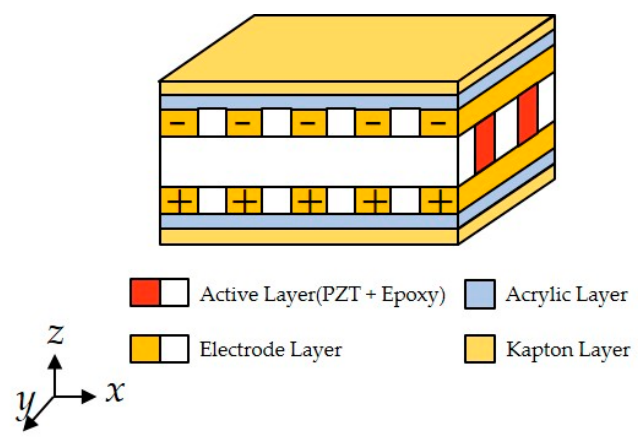

(b)

Figure 1. The structure chart of the macro fiber composite (MFC). (a) The P1 type of MFC; (b) The P2 type of MFC.

By ignoring the $x y$ in-plane shear deformation and axial deformation in the direction of the $z$ axis, the constitutive relation between the P1 and P2 types of MFC can be simplified as [26]:

$$
\begin{gathered}
{\left[\begin{array}{c}
\sigma_{x} \\
\sigma_{y} \\
\tau_{y z} \\
\tau_{z x}
\end{array}\right]=\left[\begin{array}{cccc}
Q_{11}^{E} & Q_{12}^{E} & 0 & 0 \\
Q_{21}^{E} & Q_{22}^{E} & 0 & 0 \\
0 & 0 & Q_{55}^{E} & 0 \\
0 & 0 & 0 & Q_{66}^{E}
\end{array}\right]\left[\begin{array}{c}
\varepsilon_{x}-d_{33} E_{x} \\
\varepsilon_{y}-d_{31} E_{x} \\
\gamma_{y z} \\
\gamma_{y z}
\end{array}\right],} \\
{\left[\begin{array}{c}
\sigma_{x} \\
\sigma_{y} \\
\tau_{y z}
\end{array}\right]=\left[\begin{array}{cccc}
Q_{11}^{E} & Q_{12}^{E} & 0 & 0 \\
Q_{21}^{E} & Q_{22}^{E} & 0 & 0 \\
0 & 0 & Q_{55}^{E} & 0 \\
0 & 0 & 0 & Q_{66}^{E}
\end{array}\right]\left[\begin{array}{c}
\varepsilon_{x}-d_{31} E_{z} \\
\varepsilon_{y}-d_{32} E_{z} \\
\gamma_{y z} \\
\gamma_{y z}
\end{array}\right],} \\
{[Q]=\left[\begin{array}{cccc}
\frac{E_{p x}}{1-v_{p}^{2}} & \frac{v_{p} E_{p x}}{1-v_{p}^{2}} & 0 & 0 \\
\frac{v_{p} E_{p y}}{1-v_{p}^{2}} & \frac{E_{p y}}{1-v_{p}^{2}} & 0 & 0 \\
0 & 0 & G_{p} & 0 \\
0 & 0 & 0 & G_{p}
\end{array}\right],}
\end{gathered}
$$

where $E_{p x}$ and $E_{p y}$ are the elasticity modulus of the MFC along the directions of the $x$ axis and $y$ axis; $v_{p}$ is the Poisson's ratio of the MFC; $G_{p}$ is the shear modulus of the MFC; in the P1 type MFC, $d_{31}$ is the strain of the MFC produced along the direction of the $y$ axis in the electric field action in the direction of the $x$ axis; $d_{33}$ is the strain of the MFC produced along the direction of the $x$ axis in the electric field action in the direction of the $x$ axis. In the P2 type MFC, $d_{31}$ is the strain of MFC produced along the direction of $z$ axis in the electric field action in the direction of $x$ axis; $d_{32}$ is the strain of the MFC produced along the direction of the $z$ axis in the electric field action in the direction of the $y$ axis; $E_{x}$ is the electric field intensity in the direction of the $x$ axis. $E_{z}$ is the electric field intensity in the direction of the $z$ axis.

\section{The Actuating Equation of an MFC Laminated Plate Structure}

The laminated plate is made by single-face stickup piece of MFC, whose size is shown in Figure 2, with the original point of coordinate axes in the central position of the plate. $l_{1}$ and $l_{2}$ are the MFC's effective width and length; $R$ is the distance between the plate midline and the upper surface of the $\mathrm{MFC} ; h_{p}$ is the thickness of the MFC; the thickness of the plate is $2 r$, the elasticity modulus is $E_{s}$, the shear elasticity is $G_{S}$, Poisson's ratio is $v_{S}$. 


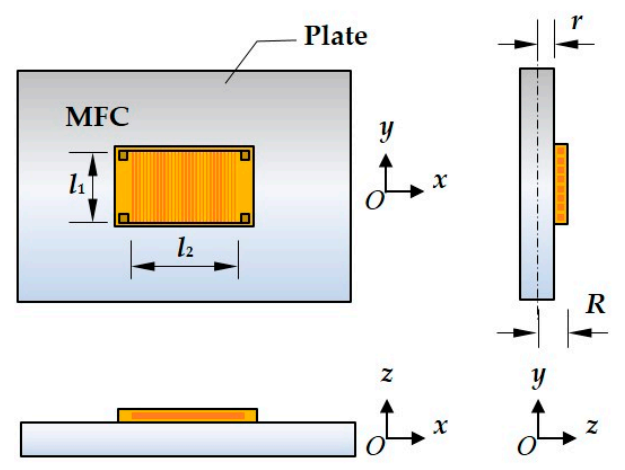

Figure 2. MFC laminated plate structure.

The action of voltage will create axial, bending and shear deformations of the MFC attached to the plate's surface. The actuating force caused by the deformations will be transmitted through the layer sticking up between the MFC and the plate. To consider the influence of the shear deformation of the MFC laminated plate on the MFC actuating force, SSDT [32] is adopted to deduce the MFC actuating force formula. Considering that differences exist in material parameters between the MFC and the plate, deformation compatibility and stress balance should be ensured between them, global-local displacement distribution functions are introduced to establish the stress balance equation of MFC laminated plate, and then, according to the equal shear force between the MFC and the plate, the system of stress partial differential equations is simplified and solved to obtain the interfacial shear force between the MFC and the plate structure and the actuating force and actuating bending moment of the MFC.

The following hypothesis is assumed for the MFC actuating force calculation: (1) the MFC and plate structure are entirely adhered without considering the thickness of the stickup layer [21]; (2) the MFC curvature along the directions of the $x$ and $y$ axes can be viewed as constant [19]; (3) no shear deformation exists at the midline position of the $x y$ in-plane MFC laminated plate [33].

According to the SSDT proposed by Touratier [25], displacement of the MFC laminated plate is composed of axial displacement, displacement created by bending deformation and by shear deformation. Suppose that the displacement $w(x, y, z)$ in the normal direction of the structure has nothing to do with $z$, then the displacement and strain on the MFC laminated plate can be indicated as:

$$
\begin{gathered}
u(x, y, z)=u_{0}(x, y)-z \frac{\partial w(x, y)}{\partial x}+\psi_{x}(x, y) f_{x}(z) \\
v(x, y, z)=v_{0}(x, y)-z \frac{\partial w(x, y)}{\partial y}+\psi_{y}(x, y) f_{y}(z) \\
w(x, y, z)=w(x, y)
\end{gathered}
$$

where $u_{0}(x, y)$ and $v_{0}(x, y)$ are the displacements in the mid-place position along the $x$ and $y$ axes; $w(x, y)$ is the lateral displacement of the plate; $\psi_{x}(x, y)$ and $\psi_{y}(x, y)$ are the shear deformations of the cross section along the $x$ and $y$ axes; $f_{x}(z)$ and $f_{y}(z)$ are the displacement distribution functions along the thickness in the $x$ and $y$ directions.

Due to different material properties of the MFC and the plate structure, global-local displacement distribution functions are introduced to ensure deformation compatibility and stress balance between them, among which $f_{g}(z)$ is the global displacement distribution function and $f_{l}(z)$ is the local displacement distribution function. Consequently, the sectional global-local displacement distribution functions of MFC laminated plate are:

$$
\begin{gathered}
f_{x}(z)=f_{y}(z)=f_{g}(z)+f_{l}(z), f_{g}(z)=\left(\frac{R+r}{\pi}\right) \sin \left(\frac{\pi z}{R+r}\right) \\
f_{l}(z)=\left\{\begin{array}{c}
a_{1} \xi_{1}+b_{1} \xi_{1}{ }^{2}, z \in[R, r] \\
a_{2} \xi_{2}+b_{2} \xi_{2}{ }^{2}, z \in[r,-r]
\end{array},\right.
\end{gathered}
$$


where $\xi_{1}$ and $\xi_{2}$ are local coordinates of the section.

$$
\xi_{1}=\frac{2 z-R-r}{R-r}, \xi_{2}=\frac{z}{r},
$$

According to deformation compatibility and stress balance, the shear stress between the upper surface of the MFC and the lower surface of the plate is 0 , and the displacement of the raised layer between the MFC and the plate structure should remain continuous and their shear stresses should be equal. The relevant parameters of the local displacement distribution function can be obtained from the above-mentioned boundary conditions:

$$
\begin{gathered}
a_{1}=\frac{(r-R)\left(\left(3 G_{p}-4 G_{s}\right) r \cos \left(\frac{\pi r}{R+r}\right)+\left(3 G_{p} r+G_{s}(R-r)\right) \cos \left(\frac{\pi R}{R+r}\right)\right)}{12 G_{p} r+6 G_{s}(R-r)} \\
a_{2}=\frac{r\left(\left(-7 G_{p} r+3 G_{p} R+6 G_{s}(r-R)\right) \cos \left(\frac{\pi r}{R+r}\right)+G_{p}(R-r) \cos \left(\frac{\pi R}{R+r}\right)\right)}{12 G_{p} r+6 G_{s}(R-r)} \\
b_{1}=\frac{(R-r)\left(\left(3 G_{p}-4 G_{s}\right) r \cos \left(\frac{\pi r}{R+r}\right)+\left(-3 G_{p} r+2 G_{s}(r-R)\right) \cos \left(\frac{\pi R}{R+r}\right)\right)}{24 G_{p} r+12 G_{s}(R-r)} \\
b_{2}=\frac{G_{p} r\left((5 r+3 R) \cos \left(\frac{\pi r}{R+r}\right)+(R-r) \cos \left(\frac{\pi R}{R+r}\right)\right)}{24 G_{p} r+12 G_{s}(R-r)}
\end{gathered} .
$$

To establish the relationship between shearing strain and displacement, the MFC is used as the object to obtain its shearing strain in $x z$ plane with Equation (5):

$$
\begin{gathered}
\gamma_{z x}(x, y, z)=\frac{\partial u}{\partial z}+\frac{\partial w}{\partial x}=\frac{1}{\Lambda_{p x}}\left(u_{R}-u_{r}+\int_{r}^{R} \frac{\partial w(x, y)}{\partial x} d z\right) \frac{d f_{x}(z)}{d z} \\
\Lambda_{p x}=\Lambda_{p y}=\frac{R+r}{\pi}\left(\sin \left(\frac{\pi R}{R+r}\right)-\sin \left(\frac{\pi r}{R+r}\right)\right)+2 a_{1}
\end{gathered}
$$

where $u_{R}$ and $u_{r}$ represent the displacements at the positions of $z=R$ and $z=r$ along the direction of the $x$ axis.

The stress balance equation of the upper surface of the MFC is obtained according to the stress balance of the MFC:

$$
\begin{gathered}
\frac{d \sigma_{p x, R}}{d x}+\frac{G_{p} \Omega_{p x, R}}{\Lambda_{p x}}\left(u_{R}-u_{r}+\int_{r}^{R} \frac{\partial w(x, y)}{\partial x} d z\right)=0, \\
\Omega_{p x, R}=\Omega_{p y, R}=\frac{8 b_{1}}{(R-r)^{2}}-\frac{\pi}{R+r} \sin \left(\frac{\pi R}{R+r}\right),
\end{gathered}
$$

where $\sigma_{p x, R}$ is the normal stress of the upper surface of the MFC along the direction of the $x$ axis.

Similarly, the shearing strain of the plate structure in the $x z$ plane is:

$$
\begin{aligned}
\gamma_{z x}(x, y, z) & =\frac{1}{\Lambda_{s x}}\left(u_{r}-u_{-r}+\int_{-r}^{r} \frac{\partial w(x, y)}{\partial x} d z\right) \frac{d f_{x}(z)}{d z}, \\
\Lambda_{s x} & =\Lambda_{s y}=2 a_{2}+\frac{R+r}{\pi} \sin \left(\frac{\pi r}{R+r}\right)
\end{aligned}
$$

where $u_{-r}$ represents the displacement at the position of $z=-r$ along the direction of the $x$ axis.

Similarly, the stress balance equation of the upper surface of the plate can be obtained with its upper surface as the object:

$$
\begin{gathered}
\frac{d \sigma_{s x, r}}{d x}+\frac{G_{s} \Omega_{s x, r}}{\Lambda_{s x}}\left(u_{r}-u_{-r}+\int_{-r}^{r} \frac{\partial w(x, y)}{\partial x} d z\right)=0, \\
\Omega_{s x, r}=\Omega_{s y, r}=-\frac{2 b_{2}}{r^{2}}+\frac{\pi}{R+r} \sin \left(\frac{\pi r}{R+r}\right),
\end{gathered}
$$

where $\sigma_{s x, R}$ is the normal stress of the upper surface of the plate along the direction of the $x$ axis. 
According to the equal shear stress on the raised interface, Equations (5), (9) and (13) are combined:

$$
\begin{aligned}
& \left.G_{p}\left(\psi_{x}(x, y)+\frac{\partial w(x, y)}{\partial x}\right) \frac{d f_{x}(z)}{d z}\right|_{z=r+}=\left.G_{s}\left(\psi_{x}(x, y)+\frac{\partial w(x, y)}{\partial x}\right) \frac{d f_{x}(z)}{d z}\right|_{z=r-} \\
& \frac{G_{p}}{\Lambda_{p x}}\left(u_{R}-u_{r}+\int_{r}^{R} \frac{\partial w(x, y)}{\partial x} d z\right) \frac{d f_{x}(z)}{d z}=\frac{G_{s}}{\Lambda_{s x}}\left(u_{r}-u_{-r}+\int_{-r}^{r} \frac{\partial w(x, y)}{\partial x} d z\right) \frac{d f_{x}(z)}{d z}
\end{aligned}
$$

where $z=r+$ represents the lower surface of the MFC; $z=r$ - represents the upper surface of the plate structure.

Equation (17) is simplified as:

$$
u_{r}=\frac{\Lambda_{s x}}{\Lambda_{p x}}\left(u_{R}-u_{r}+\int_{r}^{R} \frac{\partial w(x, y)}{\partial x} d z\right)+u_{-r}-\int_{-r}^{r} \frac{\partial w(x, y)}{\partial x} d z .
$$

Equation (18) is substituted into Equation (15) to eliminate the displacement $u_{-r}$ of the lower surface of the plate along the direction of $x$ axis, obtaining:

$$
\frac{d \sigma_{s x, r}}{d x}+\frac{G_{s} \Omega_{s x, r}}{\Lambda_{p x}}\left(u_{R}-u_{r}+\int_{r}^{R} \frac{\partial w(x, y)}{\partial x} d z\right)=0 .
$$

According to the stress-strain relationship, Equations (11) and (19) are translated into the system of strain partial differential equations of the upper surface of the MFC and the upper surface of the plate:

$$
\begin{gathered}
\frac{d^{2} \varepsilon_{s x, r}}{d x^{2}}+\frac{G_{s} \Omega_{s x, r} h_{p}}{Q_{s} \Lambda_{p x}}\left(\frac{\varepsilon_{p x, R}-\varepsilon_{s x, r}}{h_{p}}+\frac{\partial^{2} w(x, y)}{\partial x^{2}}\right)=0 \\
\frac{d^{2} \varepsilon_{p x, R}}{d x^{2}}+\frac{G_{p} \Omega_{p x, R} h_{p}}{Q_{11}^{E} \Lambda_{p x}}\left(\frac{\varepsilon_{p x, R}-\varepsilon_{s x, r}}{h_{p}}+\frac{\partial^{2} w(x, y)}{\partial x^{2}}\right)=0
\end{gathered},
$$

where $Q_{s}=\frac{E_{s}}{1-v_{s}^{2}}$.

According to the piezoceramics actuating force formula put forward by Crawley et al. [19], the mutation of MFC stress-strain along the direction of the $x$ axis appears only at the border position of the piezoelectric material, while its stress-strain at other positions almost remains constant. A proportional relationship exists between the MFC curvature and the normal strain distribution of the cross-section, and the normal strain distribution except that at border position remains constant. Thus, supposing that the curvature of the MFC laminated plate structure along the direction of the $x$ axis is constant, then it is obtained by Equation (20):

$$
\frac{d^{4} \varepsilon_{s x, r}}{d x^{4}}+\frac{G_{s} \Omega_{s x, r}}{Q_{s} \Lambda_{p x}}\left(\varepsilon_{p x, R}^{\prime \prime}-\varepsilon_{s x, r}^{\prime \prime}\right)=0, \frac{d^{4} \varepsilon_{p x, R}}{d x^{4}}+\frac{G_{p} \Omega_{p x, R}}{Q_{11}^{E} \Lambda_{p x}}\left(\varepsilon_{p x, R}^{\prime \prime}-\varepsilon_{s x, r}^{\prime \prime}\right)=0 .
$$

Because no normal stress at the border position of the upper surface of the MFC and the normal strain along the direction of $x$ axis is symmetric around the $y$ axis, the following is obtained:

$$
\varepsilon_{p x, R}=X_{1} \cosh (\alpha x)+Y_{1}, \varepsilon_{s x, r}=X_{2} \cosh (\alpha x)+Y_{2},
$$

where

$$
\alpha=\sqrt{\frac{G_{s} \Omega_{s x, r}}{Q_{s} \Lambda_{p x}}-\frac{G_{p} \Omega_{p x, R}}{Q_{11}^{E} \Lambda_{p x}}} .
$$

Equation (22) is the general solution form of the strain distribution of the upper surface of the MFC. To calculate the strain distribution of the upper surface of the MFC further, the strain at the midline position and the border position of the upper surface of the MFC should be obtained. Since the raised position between the MFC and the plate is symmetrical and no shear deformation is at the midline position, $\psi_{x}(0, y)$ in Equation (5) is 0 and the midline position is shown in Figure 3. With Equation (5), the strain $\varepsilon_{x, c}$ and $\varepsilon_{y, c}$ can be obtained at the midline position of the MFC laminated plate 
along the directions of the $x$ and $y$ axes, as shown in Equation (24). Then, according to the mechanical equilibrium equation of the section at the midline position, membrane strain $\varepsilon_{x 0}^{\prime}$ and $\varepsilon_{y 0}^{\prime}$, and curvature $\gamma_{x}$ and $\gamma_{y}$ can be obtained to calculate the strain $\varepsilon_{p x, c}$ and $\varepsilon_{p y, c}$ at the midline position on the upper surface of the MFC along the directions of the $x$ and $y$ axes [24], as is shown in Equation (25). For the P1 type MFC, $\varepsilon_{x 0}=d_{33} E_{x}, \varepsilon_{y 0}=d_{31} E_{x}$; for the P2 type MFC, $\varepsilon_{x 0}=d_{31} E_{z}, \varepsilon_{y 0}=d_{32} E_{z}$.

$$
\begin{gathered}
\varepsilon_{x, c}=\varepsilon_{x 0}^{\prime}+z \gamma_{x}, \varepsilon_{y, c}=\varepsilon_{y 0}^{\prime}+z \gamma_{y}, \\
\varepsilon_{p x, c}=\varepsilon_{x 0}^{\prime}+\gamma_{x} R, \varepsilon_{p y, c}=\varepsilon_{y 0}^{\prime}+\gamma_{y} R,
\end{gathered}
$$

where,

$$
\begin{gathered}
\gamma_{x}=\frac{v_{p}\left(B Q-A R^{\prime}\right)\left(T^{2}-S U^{\prime}\right)\left(\varepsilon_{y 0}+v_{p} \varepsilon_{x 0}\right)+\left(C U^{\prime}-D T\right)\left(Q^{2}-P R^{\prime}\right)\left(\varepsilon_{x 0}+v_{p} \varepsilon_{y 0}\right)}{\left(v_{p}^{2}-1\right)\left(Q^{2}-P R^{\prime}\right)\left(T^{2}-S U^{\prime}\right)} \\
\varepsilon_{x 0}^{\prime}=\frac{v_{p}(A Q-B P)\left(T^{2}-S U^{\prime}\right)\left(\varepsilon_{y 0}+v_{p} \varepsilon_{x 0}\right)+(D S-C T)\left(Q^{2}-P R^{\prime}\right)\left(\varepsilon_{x 0}+v_{p} \varepsilon_{y 0}\right)}{\left(v_{p}^{2}-1\right)\left(Q^{2}-P R^{\prime}\right)\left(T^{2}-S U^{\prime}\right)} \\
\gamma_{y}=\frac{\left(A R^{\prime}-B Q\right)\left(T^{2}-S U^{\prime}\right)\left(\varepsilon_{y 0}+v_{p} \varepsilon_{x 0}\right)+v_{p}\left(D T-C U^{\prime}\right)\left(Q^{2}-P R^{\prime}\right)\left(\varepsilon_{x 0}+v_{p} \varepsilon_{y 0}\right)}{\left(v_{p}^{2}-1\right)\left(Q^{2}-P R^{\prime}\right)\left(T^{2}-S U^{\prime}\right)} \\
\varepsilon_{y 0}^{\prime}=\frac{(B P-A Q)\left(T^{2}-S U^{\prime}\right)\left(\varepsilon_{y 0}+v_{p} \varepsilon_{x 0}\right)+v_{p}(C T-D S)\left(Q^{2}-P R^{\prime}\right)\left(\varepsilon_{x 0}+v_{p} \varepsilon_{y 0}\right)}{\left(v_{p}^{2}-1\right)\left(Q^{2}-P R^{\prime}\right)\left(T^{2}-S U^{\prime}\right)} \\
A=\frac{\left(R^{2}-r^{2}\right) E_{p y}}{2\left(1-v_{p}^{2}\right)}, B=\frac{h_{p} E_{p y}}{1-v_{p}^{2}}, C=\frac{A E_{p x}}{E_{p y}}, D=\frac{B E_{p x}}{E_{p y}}, P=\frac{2 r^{3} E_{s}+\left(R^{3}-r^{3}\right) E_{p y}}{3\left(1-v_{p}^{2}\right)} \\
Q=\frac{E_{p y}\left(R^{2}-r^{2}\right)}{2\left(1-v_{p}^{2}\right)}, R^{\prime}=\frac{2 r E_{s}+h_{p} E_{p y}}{1-v_{p}^{2}}, S=\frac{2 r^{3} E_{s}+\left(R^{3}-r^{3}\right) E_{p x}}{3\left(1-v_{p}^{2}\right)} \\
T=\frac{E_{p x}\left(R^{2}-r^{2}\right)}{2\left(1-v_{p}^{2}\right)}, U^{\prime}=\frac{2 r E_{s}+h_{p} E_{p x}}{1-v_{p}^{2}}
\end{gathered}
$$
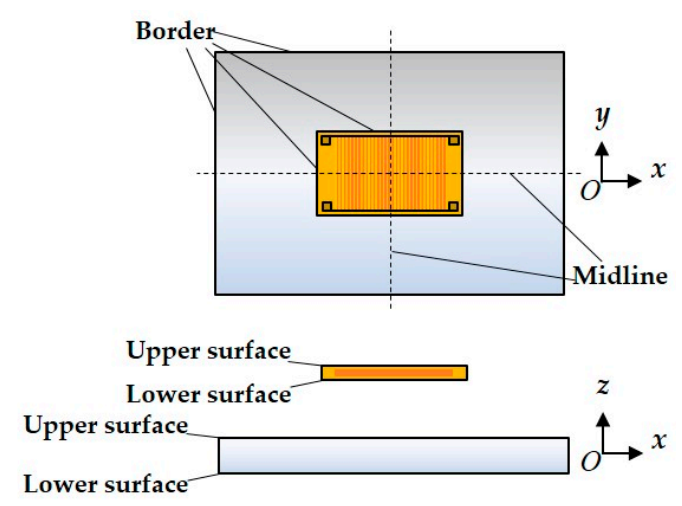

Figure 3. Boundary position of the MFC laminated plate.

Figure 4 shows the stress of the infinitesimal element at the border position on the upper surface of the MFC. Equation (22) explains that the gradient change of the strain exists along the longer border position of the infinitesimal element. Therefore, the strain $\varepsilon_{p x, e}$ and $\varepsilon_{p y, e}$ at the border position of the upper surface of the MFC can be obtained through the stress balance equation of the infinitesimal element:

$$
\begin{aligned}
& Q_{11}^{E}\left(\varepsilon_{p x, e}-\varepsilon_{x 0}\right)+Q_{12}^{E}\left(\frac{\varepsilon_{y 0} \cosh (\beta y)}{\cosh \left(0.5 \beta l_{1}\right)}+\varepsilon_{p y, c}\left(1-\frac{\cosh (\beta y)}{\cosh \left(0.5 \beta l_{1}\right)}\right)-\varepsilon_{y 0}\right)=0 \\
& Q_{21}^{E}\left(\frac{\varepsilon_{x 0} \cosh (\alpha x)}{\cosh \left(0.5 \alpha l_{2}\right)}+\varepsilon_{p y, c}\left(1-\frac{\cosh (\alpha x)}{\cosh \left(0.5 \alpha l_{2}\right)}\right)-\varepsilon_{x 0}\right)+Q_{22}^{E}\left(\varepsilon_{p y, e}-\varepsilon_{y 0}\right)=0
\end{aligned} .
$$




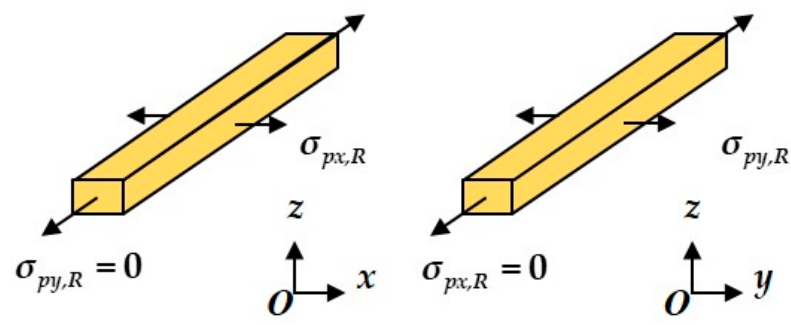

Figure 4. The stress of the infinitesimal element at the border position on the upper surface of the MFC.

The strain at the border position on the upper surface of the MFC is:

$$
\begin{aligned}
& \varepsilon_{p x, e}=\varepsilon_{x 0}+\frac{Q_{12}^{E}}{Q_{11}^{E}}\left(\varepsilon_{y 0}-\varepsilon_{p y, c}\right)\left(1-\frac{\cosh (\beta y)}{\cosh \left(0.5 \beta l_{1}\right)}\right) \\
& \varepsilon_{p y, e}=\varepsilon_{y 0}+\frac{Q_{21}^{E}}{Q_{22}^{E}}\left(\varepsilon_{x 0}-\varepsilon_{p x, c}\right)\left(1-\frac{\cosh (\alpha x)}{\cosh \left(0.5 \alpha l_{2}\right)}\right)
\end{aligned} .
$$

According to Equation (29), mutations of $\varepsilon_{p x, e}$ and $\varepsilon_{p y, e}$ exist at the two corners of the border position of the MFC in the directions of the $x$ and $y$ axes, but normal strain at other border positions remains essentially constant. Therefore, the normal strain at the border position on the upper surface of the MFC can be regarded as a constant value:

$$
\bar{\varepsilon}_{p x, e}=\varepsilon_{x 0}+\frac{Q_{12}}{Q_{11}}\left(\varepsilon_{y 0}-\varepsilon_{p y, c}\right), \bar{\varepsilon}_{p y, e}=\varepsilon_{y 0}+\frac{Q_{21}}{Q_{22}}\left(\varepsilon_{x 0}-\varepsilon_{p x, c}\right) .
$$

Equations (25) and (30) are substituted into Equation (22) to obtain the normal strain of the upper surface of the MFC along the direction of the $x$ axis:

$$
\varepsilon_{p x, R}=\frac{\bar{\varepsilon}_{p x, e} \cosh (\alpha x)}{\cosh \left(0.5 \alpha l_{2}\right)}+\varepsilon_{p x, c}\left(1-\frac{\cosh (\alpha x)}{\cosh \left(0.5 \alpha l_{2}\right)}\right) .
$$

Equations (9), (11) and (31) are combined to obtain the interfacial shear stress between the MFC and the plate structure along the direction of the $x$ axis, namely, the actuating stress of the MFC along the direction of the $x$ axis:

$$
\tau_{x}=\frac{\alpha f_{r} Q_{11}^{E} \cosh (\alpha x)}{\Omega_{p x, R} \cosh \left(0.5 \alpha l_{2}\right)}\left(\varepsilon_{p x, c}-\bar{\varepsilon}_{p x, e}\right)
$$

where,

$$
f_{r}=1-\frac{4 r^{2}}{(R+r)^{2}}+\frac{2 a_{1}-4 b_{1}}{h_{p}},
$$

In a similar way, the interfacial shear stress along the direction of the $y$ axis is obtained, namely, the actuating stress of the MFC along the direction of the $y$ axis:

$$
\tau_{y}=\frac{\beta f_{r} Q_{22}^{E} \sinh (\beta y)}{\Omega_{p y, R} \cosh \left(0.5 \beta l_{1}\right)}\left(\varepsilon_{p y, c}-\bar{\varepsilon}_{p y, e}\right),
$$

where,

$$
\beta=\sqrt{\frac{G_{s} \Omega_{s y, r}}{Q_{s} \Lambda_{p y}}-\frac{G_{p} \Omega_{p y, R}}{Q_{22}^{E} \Lambda_{p y}}}, \Omega_{s y, r}=\Omega_{s x, r}, \Lambda_{s y}=\Lambda_{s x}, \Omega_{p y, R}=\Omega_{p x, R}, \Lambda_{p y}=\Lambda_{p x} .
$$

According to Equations (25), (26) and (30), $\varepsilon_{p x, c}, \varepsilon_{p y, c}, \bar{\varepsilon}_{p x, e}$ and $\bar{\varepsilon}_{p y, e}$ all include an influence of the piezoelectric effect in the directions of the $x$ and $y$ axes, and the magnitude of the interfacial shear stress $\tau_{x}$ and $\tau_{y}$ is related to the piezoelectric effect in the directions of the $x$ and $y$ axes. According 
to Equations (32) and (34), the total actuating forces $T_{x}$ and $T_{y}$ of the MFC at the center of the MFC laminated plate structure can be obtained:

$$
T_{x}=\frac{f_{r} l_{1} Q_{11}^{E}}{\Omega_{p x, R}}\left(\varepsilon_{p x, c}-\bar{\varepsilon}_{p x, e}\right), T_{y}=\frac{f_{r} l_{2} Q_{22}^{E}}{\Omega_{p y, R}}\left(\varepsilon_{p y, c}-\bar{\varepsilon}_{p y, e}\right) .
$$

Equation (36) indicates that the MFC actuating force is related to the elasticity modulus and the thickness of the plate structure. To explore their relationship, we have analyzed the relationship between the elasticity modulus of the plate, the thickness of plate and the total actuating force of the M2814-P1 type and the M2814-P2 type of MFC along the direction of the $x$ axis under a voltage of $1500 \mathrm{~V}$, as shown in Figure 5. It shows that the total actuating force of the MFC increases with the elasticity modulus and the thickness of the beam structure at first, then its growth rate gradually slows down and tends to become stable. There is a non-linear relationship between the actuating force of the MFC, the structural elasticity modulus and the thickness.

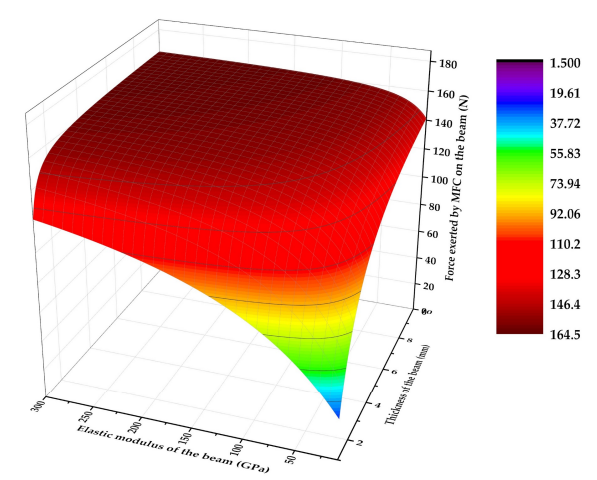

(a)

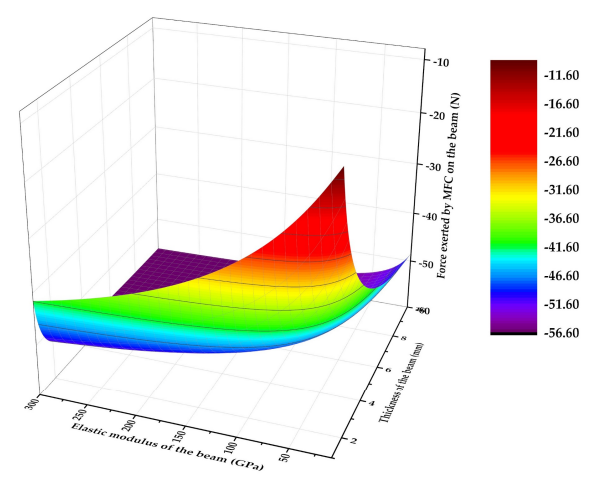

(b)

Figure 5. The relationship between the total actuating force of the MFC, thickness, and the structural elasticity modulus, in (a) the M2814-P1 type; (b) the M2814-P2 type.

A comparison has been made between the distribution formula of the mean normal stress based on SSDT and that based on CPT to analyze the distribution regularities of the mean normal stress of the MFC in MFC laminated plates. Since the normal stress at the sticking border position is 0 [16], the mean normal stress of the MFC can be obtained through the stress balance:

$$
\bar{\sigma}_{p x}=\frac{f Q_{11}^{E}\left(\bar{\varepsilon}_{x, e}-\varepsilon_{p x, c}\right)}{\Omega_{p x, R}(R-r)}\left(1-\frac{\cosh (\alpha x)}{\cosh \left(0.5 \alpha l_{2}\right)}\right), \bar{\sigma}_{p y}=\frac{f Q_{22}^{E}\left(\bar{\varepsilon}_{y, e}-\varepsilon_{p y, c}\right)}{\Omega_{p y, R}(R-r)}\left(1-\frac{\cosh (\beta y)}{\cosh \left(0.5 \beta l_{1}\right)}\right) .
$$

The mean stress of the MFC based on CPT can be described as [24]:

$$
\begin{aligned}
& \bar{\sigma}_{p x, c p t}=\frac{1}{R-r}\left(\int_{r}^{R} Q_{11}^{E}\left(\varepsilon_{x 0}^{\prime}+\gamma_{x} z-\varepsilon_{x 0}\right)+Q_{12}^{E}\left(\varepsilon_{y 0}^{\prime}+\gamma_{y} z-\varepsilon_{y 0}\right) d z\right) \\
& \bar{\sigma}_{p y, c p t}=\frac{1}{R-r}\left(\int_{r}^{R} Q_{21}^{E}\left(\varepsilon_{x 0}^{\prime}+\gamma_{x} z-\varepsilon_{x 0}\right)+Q_{22}^{E}\left(\varepsilon_{y 0}^{\prime}+\gamma_{y} z-\varepsilon_{y 0}\right) d z\right) .
\end{aligned} .
$$

By comparing Equations (37) and (38), it is proved that the MFC mean normal stress formula based on SSDT provides the distribution regularities of the MFC mean normal stress as a type of hyperbolic cosine function with consideration of shear deformation, while the one based on CPT cannot, because it only considers the stress-strain distribution of the MFC laminated plate structure along the direction of thickness, ignoring in-plane shear deformation, and cannot obtain the shear stress distribution and the normal stress distribution between the MFC and plate structure. 


\section{Example Analysis of MFC Laminated Beam Structure}

To explain the precision of the MFC actuating force formula based on SSDT, the actuating experiment and finite element analysis of the MFC laminated beam in [29] is exemplified for analysis and discussion. It adopts the M2814-P1 and M2814-P2 types of MFC, with the distance between the raised position and the fixed end being $10 \mathrm{~mm}$. The MFC laminated beam structure is shown in Figure 6, and the parameters of the P1 type and the P2 type of MFC and those of the beam's structure in are shown in Table 1. The MFC actuating force formula based on SSDT deduced in this paper is used to calculate the mean internal force distribution of the two types of MFC under voltages of 50-300 V and $60-360 \mathrm{~V}$, as shown in Figures 7 and 8.

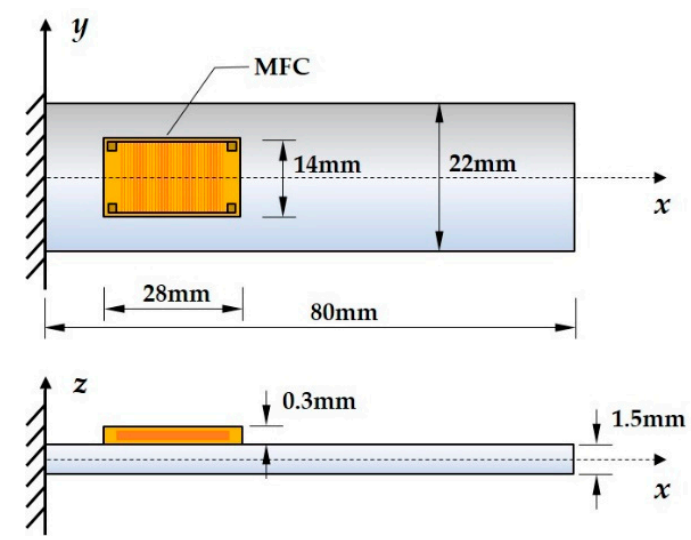

Figure 6. Model of the MFC laminated beam structure.

Table 1. Parameters of the beam structure and MFC [29].

\begin{tabular}{cccc}
\hline Material & Beam & MFC-P1 & MFC-P2 \\
\hline Elasticity modulus (GPa) & 210 & 29.4 & 30.336 \\
Shearing modulus (GPa) & 80 & 15.2 & 15.85 \\
Density (kg.m-3) & 7850 & 6.06 & 5.515 \\
Poisson's ratio & 0.31 & 5440 & 5440 \\
Piezoelectric strain constant & $/$ & 0.312 & 0.31 \\
$d_{33}(\mathrm{P} 1) / d_{31}(\mathrm{P} 2)(\mathrm{C} / \mathrm{N})$ & & 467 & -170 \\
Piezoelectric strain constant & $/$ & -210 & -100 \\
$d_{31}(\mathrm{P} 1) / d_{32}(\mathrm{P} 2)(\mathrm{C} / \mathrm{N})$ & $80 \times 22 \times 1.5$ & $28 \times 14 \times 0.3$ & $28 \times 14 \times 0.3$ \\
\hline Geometric dimension $\left(\mathrm{mm}^{3}\right)$ & & &
\end{tabular}

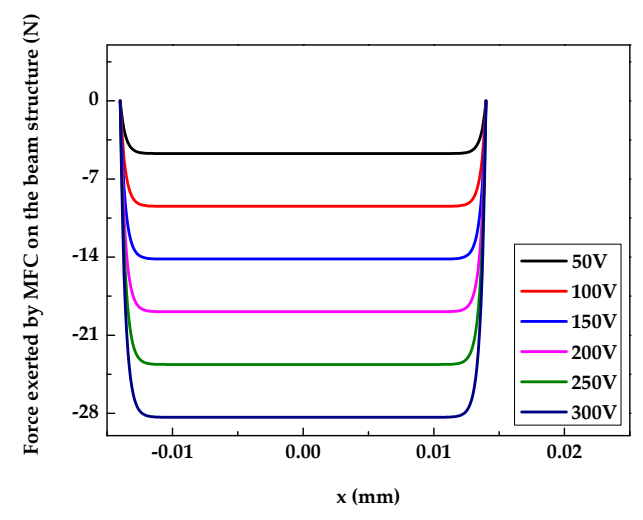

(a)

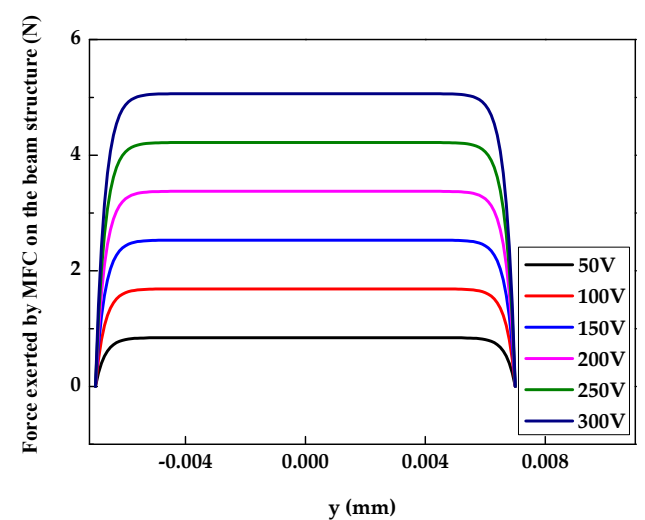

(b)

Figure 7. The mean internal force distribution of the M2814-P1 type of MFC under different voltages. (a) The direction of the $x$ axis; (b) the direction of the $y$ axis. 


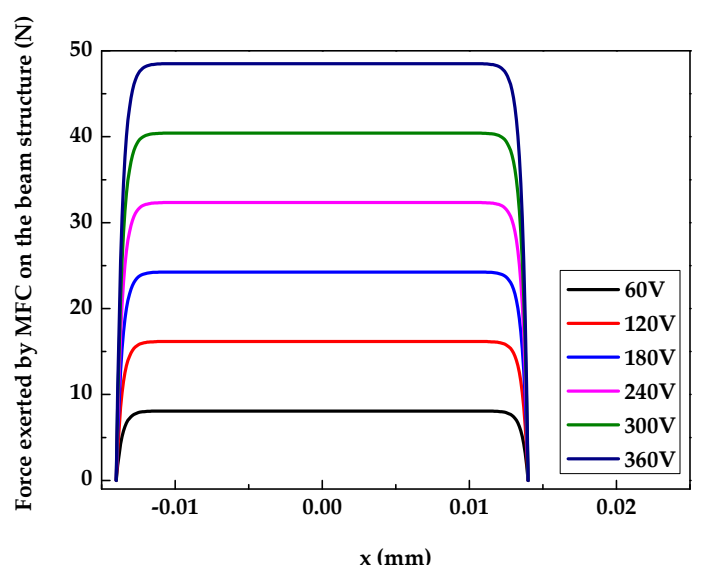

(a)

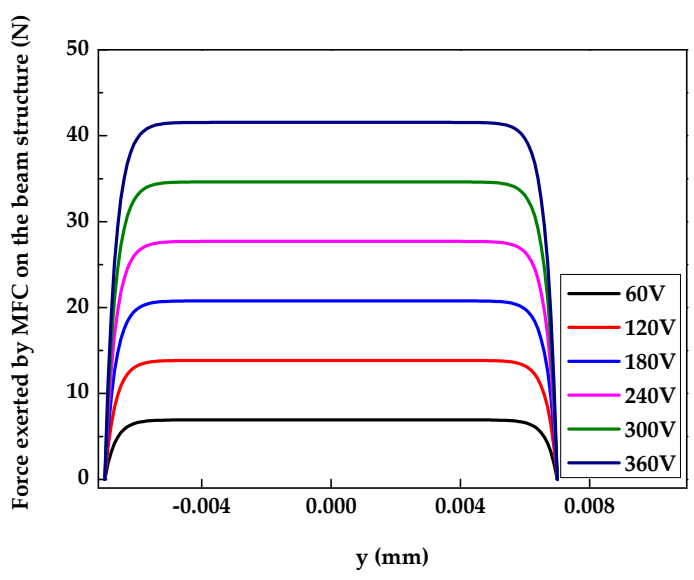

(b)

Figure 8. The mean internal force distribution of the M2814-P2 type of MFC under different voltages. (a) The direction of the $x$ axis; (b) The direction of the $y$ axis.

Figure 7 shows that under a fixed voltage, the MFC internal force along the direction of the $y$ axis is much smaller than that along the direction of the $x$ axis, meaning the direction of the $x$ axis is the main actuating direction in the P1 type of MFC. It states that the deformability of the P1 type along the direction of the $x$ axis is better than that along the direction of the $y$ axis, conforming to its characteristic deformation. In the directions of the $x$ and $y$ axes, the marginal internal force of the P1 type at the stickup position is small, the internal force increases rapidly towards the central position and tends to be stable, and the MFC in the direction of the $x$ axis takes on a compression state while the MFC in the direction of the $y$ axis takes on a tension state. Similarly, Figure 8 shows the same characteristics for the P2 type. This is created by the shear lag between the MFC and the beam structure, which reflects that at the border position of the MFC, the interfacial shear stress is much greater than that at the center and the shear stress tends to sharply declining from the positions at the border towards the center. This makes the MFC normal stress mutate at border positions, which take on hyperbolic cosine function distributions. According to the MFC's internal force distribution, its shear deformation is one of the primary factors on actuating force transmission.

In [29], actuating experiments were performed on a beam structure attached to the P1 and P2 type MFCs, respectively. Displacement and differences compared between the maximum and minimum end displacement were made in two working conditions of the same thickness and different thicknesses under changing voltages, respectively. As for the P1 MFC, the voltages of the maximum and minimum end displacement were $-500 \mathrm{~V}$ and $1500 \mathrm{~V}$; as for the P2 type, the voltages of the maximum and minimum end displacement are $240 \mathrm{~V}$ and $-120 \mathrm{~V}$. In the first place, the MFC actuating formulas based on SSDT and on CPT were used to conduct a simulation calculation on the end displacement of an MFC laminated beam of $1.5 \mathrm{~mm}$ thickness using ANSYS finite element software. In a simulation calculation of the MFC actuating force based on SSDT, MFC sub-sectional actuating forces were calculated with an effective length of $0.5 \mathrm{~mm}$ in the actuating direction, and then exerted to the corresponding sticking position through the surface effect unit in ANSYS. When the MFC actuating formula based on CPT was used, the MFC actuating force was directly exerted to the fringe nodes at the stickup position for the simulation calculation. The two simulation results are finally compared with the experimental result in [29], as shown in Figure 9. It can be seen that the simulation result from the MFC actuating force formula based on SSDT is closer to the experimental result. The maximum deviation is $2.45 \%$ between the MFC actuating force simulation results based on SSDT attached to the P1 type of MFC and the experimental results, while the maximum deviation is $9.07 \%$ between the MFC actuating simulation results based on CPT and the experimental results. A maximum deviation of $6.44 \%$ was found between the MFC actuating force simulation results based on SSDT attached to the P2 type of MFC and the 
experimental results, while a maximum deviation of $9.56 \%$ was found between the MFC actuating simulation results based on CPT and the experimental results.

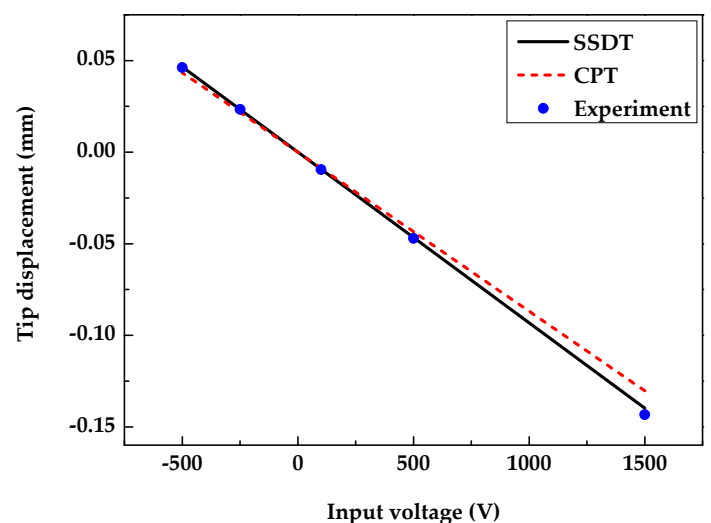

(a)

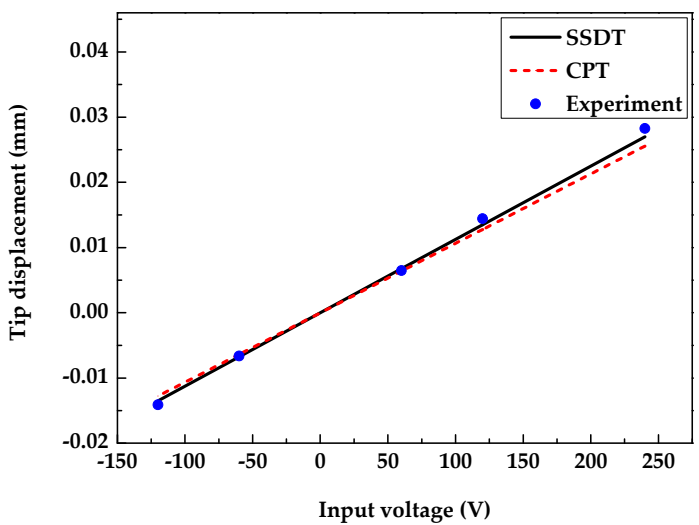

(b)

Figure 9. End displacement comparison of MFC laminated beams. (a) The P1 type of MFC; (b) the P2 type of MFC.

To make a further analysis about the actuating action of the MFC actuating force formula based on SSDT with different thicknesses deduced in this paper, a simulation and experimental comparison is made for the differences between the maximum and minimum end displacement with different thicknesses is shown in Figure 10. It can be seen that the simulation results of the MFC actuating force formula based on SSDT is closer to the experimental results. As for the P1 type of MFC, the maximum deviation is $5.78 \%$ between the MFC actuating force simulation results based on SSDT and the experimental results, while the maximum deviation is $18.03 \%$ between the MFC actuating force simulation result based on CPT and the experimental results. As for the P2 type of MFC, the maximum deviation is $7.82 \%$ between the MFC actuating force simulation results based on SSDT and the experimental results, while the maximum deviation is $13.50 \%$ between the MFC actuating force simulation results based on CPT and the experimental results. When the MFC actuating force formula based on SSDT is used, the simulation results of end displacement of the thicker beam are better than that of the thinner beam. In summary, compared with the MFC actuating force formula based on CPT, the one based on SSDT provides more precise simulation results.

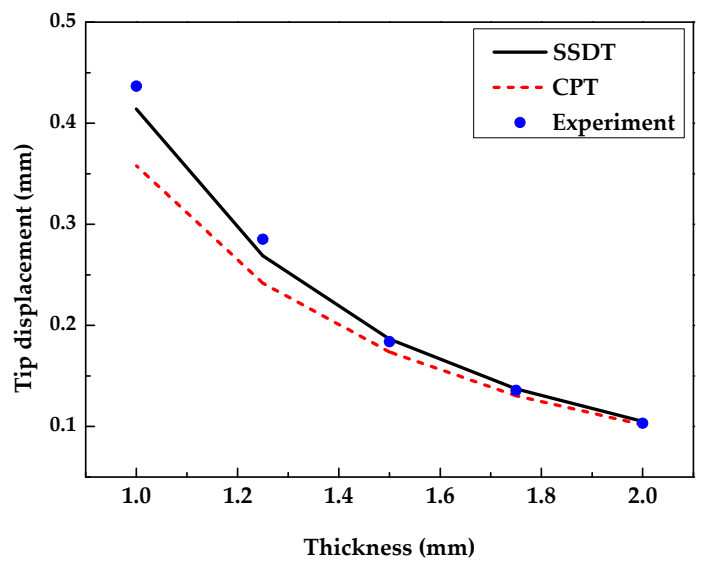

(a)

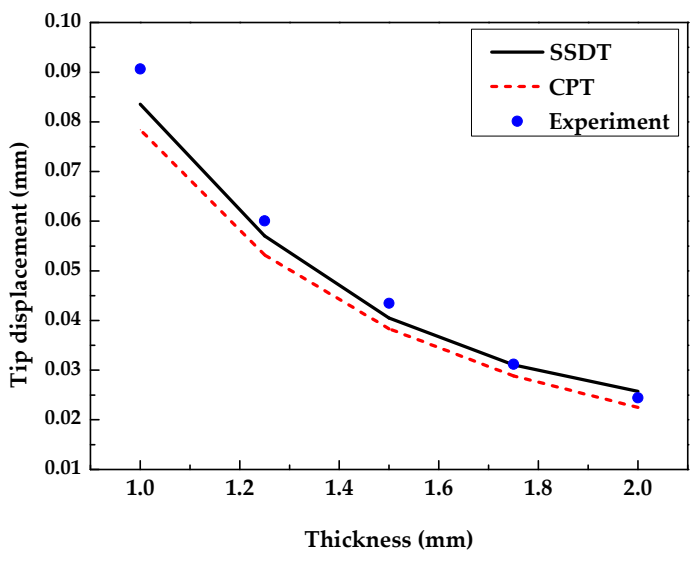

(b)

Figure 10. End displacement difference comparison of MFC laminated beams of different thicknesses. (a) The P1 type of MFC; (b) the P2 type of MFC. 


\section{Conclusions}

Based on SSDT, this paper deduced an MFC actuating force formula considering shear deformation, and drew its conclusion by verifying the simulation precision and applicability with reference to the experimental results in [29]:

1. An MFC actuating force formula based on SSDT was presented. According to the MFC actuating force formula, the interfacial shear stress between the MFC and the plate structure takes on a type of hyperbolic sine function. The influence of the elasticity modulus and thickness of the plate structure on the MFC actuating force was analyzed, which indicated that the relationship between the MFC's total actuating force, the elasticity modulus and the thickness of the plate structure appears to be non-linear, and that under fixed voltages the MFC actuating force increases with the structural elasticity modulus and thickness, and then gradually tends to become stable.

2. The MFC laminated beam in [29] served as the object to analyze the MFC mean internal force distribution under different voltages. The result is that the MFC mean internal force distribution took on a type of hyperbolic cosine function, and the MFC internal force was smaller at the sticking border position, then increased rapidly in the center and tended to become stable. As for the P1 type of MFC, the MFC actuating force in the direction of the $x$ axis is greater than that of the $y$ axis; as for the P2 type of MFC, the MFC actuating forces are close in the directions of the $x$ and $y$ axes.

3. A simulation analysis was made for the two conditions in [29], and the comparison was conducted between the simulation results and the experimental results, which showed the simulation results of end displacement from the MFC actuating force formula based on SSDT were better than those based on CPT. This proves that with greater precision and applicability, the MFC actuating force formula based on SSDT can be further popularized into the areas of MFC vibration reduction and actuation for beam-plate structures.

Author Contributions: Conceptualization, J.Z. and J.T.; Formal analysis, J.Z.; Methodology, J.Z. and J.T.; Validation, J.Z. and J.T.; Data curation, Z.L., K.G. and H.X.; Writing-original draft, J.Z.

Funding: This research was funded by the Natural Science Foundation of Hubei Province, grant number 2016CFA020; the Project of Ministry of Science and Technology, grant number 2018YFC0705601, the Fundamental Research Funds for the Central Universities, grant number 2019-YB-024.

Conflicts of Interest: The authors declare no conflict of interest.

\section{References}

1. Prasath, S.S.; Arockiarajan, A. Effective electromechanical response of macro-fiber composite (MFC): Analytical and numerical models. Int. J. Mech. Sci. 2013, 77, 98-106. [CrossRef]

2. Bent, A.A.; Hagood, N.W. Piezoelectric Fiber Composite with Interdigitated Eletrodes. J. Intell. Mater. Syst. Struct. 1997, 8, 903-919. [CrossRef]

3. Park, J.S.; Kim, J.H. Analytical development of single crystal Macro Fiber Composite actuators for active twist rotor blades. Smart Mater. Struct. 2005, 14, 745-753. [CrossRef]

4. Wilkie, W.K.; Bryant, R.G.; High, J.W.; Fox, R.L.; Hellbaum, R.F.; Jalink, A., Jr.; Little, B.D.; Mirick, P.H. Low-Cost Piezocomposite Actuator for Structural Control Applications. In Proceedings of the SPIE 7th Annual International Symposium on Smart Structures and Materials, Newport Beach, CA, USA, 6-8 March 2000.

5. Dano, M.L.; Julliere, B. Active control of thermally induced distortion in composite structures using Macro Fiber Composite actuators. Smart Mater. Struct. 2007, 16, 2315-2322. [CrossRef]

6. Kim, H.S.; Sohn, J.W.; Choi, S.B. Vibration Control of a Cylindrical Shell Structure Using Macro Fiber Composite Actuators. Mech. Based Des. Struct. 2011, 39, 491-506. [CrossRef]

7. Sohn, J.W.; Choi, S.B.; Kim, H.S. Vibration control of smart hull structure with optimally placed piezoelectric composite actuators. Int. J. Mech. Sci. 2011, 53, 647-659. [CrossRef] 
8. Sohn, J.W.; Choi, S.B. Active vibration control of a cylindrical structure using flexible piezoactuators: Experimental work in air and water environments. Smart Mater. Struct. 2014, 23, 117002. [CrossRef]

9. Zhang, S.Q.; Li, Y.X.; Schmidt, R. Modeling and simulation of macro-fiber composite layered smart structures. Compos. Part B Eng. 2015, 126, 89-100. [CrossRef]

10. Wang, X.; Zhou, W.; Wu, Z.; Wu, W. Optimal unimorph and bimorph configurations of piezocomposite actuators for bending and twisting vibration control of plate structures. J. Intell. Mater. Syst. Struct. 2018, 29, 1685-1696. [CrossRef]

11. Zhou, J.; Xu, M.; Yang, Z. Aeroelastic stability analysis of curved composite panels with embedded Macro Fiber Composite actuators. Compos. Struct. 2019, 208, 725-734. [CrossRef]

12. Gao, L.; Lu, Q.; Fei, F.; Liu, L.; Liu, Y.; Leng, J. Active vibration control based on piezoelectric smart composite. Smart Mater. Struct. 2013, 22, 125032. [CrossRef]

13. Li, L.; Xue, Z.; Li, C. Actuation Performance of Active Composite Beams with Delamination. Sens. Actuators A Phys. 2016, 249, 131-140. [CrossRef]

14. Ma, G.; Xu, M.; An, Z.; Wu, C. Active vibration control of an axially moving cantilever structure using MFC. Int. J. Appl. Electromagn. Mech. 2016, 52, 967-974. [CrossRef]

15. Dong, Z.; Farla, C.; Hromčík, M.; Pluymers, B.; Šebek, M.; Desmet, W. Equivalent force modeling of macro fiber composite actuators integrated into nonhomogeneous composite plates for dynamic applications. Smart Mater. Struct. 2017, 26, 095040. [CrossRef]

16. Sun, X.; Dai, Q.; Bilgen, O. Design and simulation of Macro-Fiber composite based serrated microflap for wind turbine blade fatigue load reduction. Mater. Res. Express 2018, 5, 055505. [CrossRef]

17. Crawley, E.F.; Luis, J. Use of Piezoelectric Actuators as Elements of Intelligent Structures. AIAA J. 1987, 25, 1373-1385. [CrossRef]

18. Crawley, E.F.; Anderson, E.H. Detailed Models of Piezoceramic Actuation of Beams. J. Intell. Mater. Syst. Struct. 1990, 1, 4-25. [CrossRef]

19. Sonti, V.R.; Kim, S.J.; Jones, J.D. Equivalent forces and wavenumber spectra of shaped piezoelectric actuators. J. Sound Vib. 1995, 187, 111-131. [CrossRef]

20. Deraemaeker, A.; Tondreau, G.; Bourgeois, F. Equivalent loads for two-dimensional distributed anisotropic piezoelectric transducers with arbitrary shapes attached to thin plate structures. J. Acoust. Soc. Am. 2011, 129, 681-690. [CrossRef]

21. Im, S.; Atluri, S.N. Effects of a Piezo-Actuator on a Finitely Deformed Beam Subjected to General Loading. AIAA J. 1989, 27, 1801-1807. [CrossRef]

22. Sayyad, A.S.; Shinde, B.M.; Ghugal, Y.M. Thermoelastic bending analysis of laminated composite plates according to various shear deformation theories. Open Eng. 2015, 5, 18-30. [CrossRef]

23. Shimpi, R.P.; Patel, H.G. A two variable refined plate theory for orthotropic plate analysis. Int. J. Solids Struct. 2006, 43, 6783-6799. [CrossRef]

24. Ghugal, Y.M.; Sayyad, A.S. A Static Flexure of Thick Isotropic Plates Using Trigonometric Shear Deformation Theory. J. Solid Mech. 2010, 2, 79-90.

25. Touratier, M. An Efficient Standard Plate Theory. Int. J. Eng. Sci. 1991, 29, 901-916. [CrossRef]

26. Ghugal, Y.M.; Sayyad, A.S. Stress analysis of thick laminated plates using trigonometric shear deformation theory. Int. J. Appl. Mech. 2013, 5, 1-23. [CrossRef]

27. Moheimani, S.O.R.; Fleming, A.J. Piezoelectric Transducers for Vibration Control and Damping, 1st ed.; Springer: London, UK, 2006; pp. 23-29.

28. Zhang, C.; Zhang, X.; Xu, M.; Luo, Y. Active control of honeycomb sandwich plate using MFC piezoelectric actuators. Int. J. Appl. Electromagn. 2014, 45, 83-91. [CrossRef]

29. Pandey, A.; Arockiarajan, A. Actuation performance of macro-fiber composite (MFC): Modeling and experimental studies. Int. J. Mech. Sci. 2016, 248, 114-129. [CrossRef]

30. Qin, Q.H. Green's Function and Boundary Elements in Multifield Materials, 1st ed.; Elsevier Science Ltd.: Oxford, UK, 2007; pp. 23-29.

31. Qin, Q.H. Advanced Mechanics of Piezoelectricity, 1st ed.; Springer: Berlin/Heidelberg, Germany, 2007; pp. $23-29$.

32. Ghugal, Y.M.; Kulkarni, S.K. Flexural analysis of cross-ply laminated plates subjected to nonlinear thermal and mechanical loadings. Acta Mech. 2013, 224, 675-690. [CrossRef] 
33. Kapuria, S.; Sharma, B.N.; Arockiarajan, A. Dynamic Shear-Lag Model for Stress Transfer in Piezoelectric Transducer Bonded to Plate. AIAA J. 2019, 57, 1-11. [CrossRef] 